University of Nebraska - Lincoln

DigitalCommons@University of Nebraska - Lincoln

2008

\title{
Impact of Solvent Quality on the Density Profiles of Looped Triblock Copolymer Brushes by Neutron Reflectivity
}

\section{Measurements}

Zhenyu Huang

Jose Alonzo

Ming Liu

Haining Ji

Fang Yin

See next page for additional authors

Follow this and additional works at: https://digitalcommons.unl.edu/usdoepub

Part of the Bioresource and Agricultural Engineering Commons

Huang, Zhenyu; Alonzo, Jose; Liu, Ming; Ji, Haining; Yin, Fang; Smith, Grant D.; Mays, Jimmy W.; Kilbey II, S. Michael; and Dadmun, Mark D., "Impact of Solvent Quality on the Density Profiles of Looped Triblock Copolymer Brushes by Neutron Reflectivity Measurements" (2008). US Department of Energy Publications. 75.

https://digitalcommons.unl.edu/usdoepub/75

This Article is brought to you for free and open access by the U.S. Department of Energy at DigitalCommons@University of Nebraska - Lincoln. It has been accepted for inclusion in US Department of Energy Publications by an authorized administrator of DigitalCommons@University of Nebraska - Lincoln. 


\section{Authors}

Zhenyu Huang, Jose Alonzo, Ming Liu, Haining Ji, Fang Yin, Grant D. Smith, Jimmy W. Mays, S. Michael Kilbey II, and Mark D. Dadmun 


\title{
Impact of Solvent Quality on the Density Profiles of Looped Triblock Copolymer Brushes by Neutron Reflectivity Measurements
}

\author{
Zhenyu Huang, ${ }^{\dagger}$ Jose Alonzo, ${ }^{\ddagger}$ Ming Liu, ${ }^{\dagger}$ Haining Ji, ${ }^{\dagger}$ Fang Yin,${ }^{\S}$ Grant D. Smith,, \\ Jimmy W. Mays, ${ }^{\dagger, \perp}$ S. Michael Kilbey II, ${ }^{\ddagger}$ and Mark D. Dadmun $*, \dagger, \perp$
}

Department of Chemistry, University of Tennessee, Knoxville, Tennessee 37996; Department of

Chemical and Biomolecular Engineering, Clemson University, Clemson, South Carolina 29634;

Department of Materials Science and Engineering and Department of Chemical Engineering, University

of Utah, Salt Lake City, Utah 84112; and Chemical Science Division, Oak Ridge National Laboratory, Oak Ridge, Tennessee 37831

Received September 28, 2007; Revised Manuscript Received December 19, 2007

\begin{abstract}
Preferential adsorption of poly(2-vinylpyridine)-deuterated polystyrene-poly(2-vinylpyridine) (PVP-dPS-PVP) triblock copolymers from toluene onto silicon leads to the formation of dPS loops tethered by the PVP end blocks. Using neutron reflectometry, we have determined the segment density profiles of these looped polymer brushes in toluene, a good solvent for the dPS block, and in cyclohexane at $20{ }^{\circ} \mathrm{C}$ (poor solvent), $32^{\circ} \mathrm{C}$, (near- $\Theta$ solvent), and $50{ }^{\circ} \mathrm{C}$ (marginal solvent). While the swelling behavior qualitatively agrees with that observed for singly grafted brushes, there are interesting differences in the local structural details: In a good solvent, the segment density profiles are composed of an inner parabolic region and a long, extended tail. In cyclohexane, the profiles are described by exponential decays. We ascribe these features to a novel polydispersity effect that arises due to tethering the PS loops by both ends. The results also show that the less dense layers undergo more significant changes in swollen height as solvent quality is changed and that the looped brushes of different molecular weight, asymmetry, and tethering density adhere to scaling relationships derived for lightly cross-linked polymer gels.
\end{abstract}

\section{Introduction}

The past two decades have seen a surge of interest in the study of polymer brushes. ${ }^{1-7}$ Interface modification using tethered polymer brushes is relevant across a broad range of applications, including colloid stabilization, ${ }^{8}$ adhesion,,${ }^{9-11}$ biomaterial interfaces, lubrication, ${ }^{12}$ and wetting. Much experimental, theoretical, and simulation work has been directed toward understanding the fundamental aspects of these tethered chains, including the adsorption kinetics and the conformation of tethered polymer chains at surfaces.

Because the extent and composition can be rigorously determined prior to layer formation, brushes formed by selective adsorption of block copolymers from solution onto the liquidsolid interface have attracted significant attention. ${ }^{13-23}$ Most of the work in this arena has focused on linear diblock copolymers, with limited work on triblock, ${ }^{20,21}$ star-block, or more complicated structures, such as combs. ${ }^{24}$ Interface-modifying layers made from these more complex macromolecules are interesting because the various architectures may provide the ability to alter surface properties in ways that are not feasible with linear copolymers. For instance, preferential adsorption from a selective solvent can be used to create multiply bound polymer chains - in the case of triblocks, loops can be formed and, in the case of star-blocks, domes. These looped interfacial layers may exhibit enhanced interfacial properties relative to singly tethered brushes. For example, Shull has shown that the loop configurations of an adsorbed chain exhibits stronger autophobicity than similar tail configurations. ${ }^{25}$ Additionally, Irvine et al. demonstrated that star polymers tethered by their ends (thus

\footnotetext{
$\dagger$ University of Tennessee.

$\doteqdot$ Clemson University.

$\S$ University of Utah.

${ }^{\perp}$ Oak Ridge National Laboratory.
}

creating loops) provide more effective resistance to protein adsorption than tethered linear polymers at the same grafting density. ${ }^{26,27}$

We have recently studied, using phase-modulated ellipsometry, the kinetics of preferential adsorption of a series of poly(2-vinylpyridine)-d-polystyrene-poly(2-vinylpyridine) (PVPdPS - PVP) triblock copolymers of varying block sizes and PS/ PVP ratios. ${ }^{28}$ This study shows that looped brushes are readily formed from these triblocks and reveals new insight into how the layer height and adsorbed amount evolve in time. Nevertheless, ellipsometry is only able to provide an "average" view of the looped layer structure. In the present study, we have used neutron reflectometry to examine the density profile of the looped brushes in different solvent conditions and as a function of copolymer composition in order to gain insight into the nanoscale, solvated structure of these brushes, as this has important implications for their interfacial properties.

Several theoretical ${ }^{29,30}$ and experimental ${ }^{31-33}$ investigations have examined the structure of singly tethered, linear polymer brushes. For instance, Karim et al. ${ }^{32}$ studied the structure of a chemically bound polystyrene brush in d-toluene, a good solvent for PS, and in d-cyclohexane, whose quality can be controlled by changing temperature. These studies show that the segment density profile is well-described by a parabolic function:

$$
\phi(z)=\phi_{\mathrm{s}}\left[1-\left(\frac{z}{h}\right)^{2}\right]^{\alpha}
$$

Here $\phi(z)$ is the segment density of the brush at the distance $z$ from the surface, $\phi_{\mathrm{s}}$ is the density at the surface, $h$ is the thickness of the brush, and $\alpha$ is an exponent that is determined by the solvent quality. Decreasing the solvent quality reduces the value of $\alpha$ from 1 (good solvent) to 0.5 ( $\Theta$ solvent) and lower (poor solvent). This is consistent with predictions from 
Table 1. Molecular Characteristics of Triblock Copolymers and Brushes Examined by Neutron Reflectivity ${ }^{a}$

\begin{tabular}{|c|c|c|c|c|c|c|c|}
\hline sample $^{b}$ & $M_{\mathrm{n}}(\mathrm{kg} / \mathrm{mol})$ & PS/PVP ratio & $M_{\mathrm{w}} / M_{\mathrm{n}}$ & $L_{\text {dry }}($ ellip) (nm) & $L_{\mathrm{dry}}(\mathrm{XR})(\mathrm{nm})$ & $\sigma\left(\mathrm{nm}^{-2}\right)$ & $\sigma^{*}=\sigma / \sigma_{0}$ \\
\hline D161K & 161 & $1: 1$ & 1.1 & 2.83 & 2.87 & 0.0242 & 2.89 \\
\hline D120K & 120 & $4: 1$ & 1.2 & 3.23 & & 0.0366 & 5.39 \\
\hline D170K & 170 & $4: 1$ & 1.2 & 3.63 & & 0.029 & 6.48 \\
\hline D136K & 136 & $10: 1$ & 1.2 & 3.96 & 4.5 & 0.0394 & 7.84 \\
\hline D252K & 252 & $10: 1$ & 1.1 & 4.08 & 4.3 & 0.0219 & 9.10 \\
\hline H216K & 216 & $10: 1$ & 1.2 & 3.8 & 4.02 & 0.0224 & 8.46 \\
\hline
\end{tabular}

${ }^{a} L_{\mathrm{dry}}\left(\right.$ ellip) is the dry layer thicknesses measured using ellipsometry, and $L_{\mathrm{dry}}(\mathrm{XR})$ is that obtained using X-ray reflectometry. The surface density, $\sigma$, is the number of PVP blocks (tethering points) per unit area, while $\sigma^{*}$, defined in eq 3 , characterizes the degree of overlap of the looped brushes. ${ }^{b} \mathrm{D}$ indicates PS-d8 and $\mathrm{H}$ indicates protonated PS.

self-consistent-field theory (SCF), ${ }^{30}$ Monte Carlo (MC) ${ }^{34}$ simulations, and molecular dynamics calculations. ${ }^{35}$ It should be noted that in our system the polymer chains were physically adsorbed from a selective solvent, and the resulting surface densities are smaller than those obtained through chemical bonding.

\section{Experimental Section}

Materials and Sample Preparation. A series of PVP-dPSPVP triblock copolymers and one PVP-hPS-PVP triblock copolymer were anionically synthesized as described in ref 28 . The molecular characteristics of these polymers are listed in Table 1. $\mathrm{D}$ and $\mathrm{H}$ in the sample name refer to samples with deuterated and protonated PS blocks, respectively, while the number denotes the total molecular weight of the triblock in thousands. HPLC grade toluene and cyclohexane were used as received.

Silicon wafers were precleaned in a bath of fuming $\mathrm{H}_{2} \mathrm{SO}_{4} / 30 \%$ $\mathrm{H}_{2} \mathrm{O}_{2}$ (3:1) piranha solution followed by rinsing with nanopure water and drying with a dry nitrogen stream. The brushes were prepared by immersing the wafers into copolymer solutions for over $15 \mathrm{~h}$. The concentration of the solution from which preferential adsorption took place was nominally $30 \mu \mathrm{g} / \mathrm{mL}$, which is anticipated to be below the critical micelle concentration. ${ }^{36}$ Upon removal from solution, the brush-modified wafers were immediately rinsed with toluene and dried under vacuum at $40{ }^{\circ} \mathrm{C}$ until the neutron reflectivity measurements were made. To verify that the preferentially adsorbed layers are not desorbed from the silicon surfaces during the neutron reflectivity measurements, control experiments were completed to verify that soaking brush-modified surfaces in cyclohexane at $50{ }^{\circ} \mathrm{C}$ and toluene at room temperature for extended periods of time $(\sim 12 \mathrm{~h})$ does not result in appreciable changes in the dry layer thicknesses as measured by ellipsometry.

Ellipsometry Measurements. Dry layer thickness were measured using an EL X-02C ellipsometer at a fixed angle of incidence (of $70^{\circ}$ ). The experimental protocols used for wafer cleaning consistently produced silicon oxide layer thicknesses in the range of $1.5-2.0 \mathrm{~nm}$; therefore, a constant value of $1.7 \mathrm{~nm}$ was used when determining the ellipsometric layer thickness and also when fitting the neutron reflectivity data. A refractive index of h-PS, which is 1.59 , was used for the triblock copolymers. The measured dry layer thicknesses were also used to estimate the surface density of chains.

Neutron Reflectivity Measurements. Neutron reflectivity measurements were performed on the NG7 reflectometer at the National Institute of Standards and Technology (NIST), Gaithersburg, MD, using a temperature-controlled liquid cell. The samples were kept in toluene at room temperature and in cyclohexane at 20,32, and $50{ }^{\circ} \mathrm{C}$ for at least $1 \mathrm{~h}$ before starting the measurements. For dPS in cyclohexane, these three temperatures correspond to poor, near- $\Theta$, and marginal solvent conditions, respectively. The neutron wavelength $(\lambda)$ was fixed at $4.76 \AA$, and the angle of incidence, $\theta$, was varied to give a wavevector $(q=4 \pi \sin \theta / \lambda)$ range from 0.01 to $0.1 \AA^{-1}$. During the measurements, the incident beam is reflected from the silicon oxide-brush-solvent interface and back through the silicon substrate to the detector. The neutron reflectivity $(R)$ was obtained as a function of $q$. The neutron scattering length density (SLD) profiles were obtained using the "reflfit" 37 program provided by NIST and then transformed to the density profiles for
Table 2. Density and Neutron Scattering Length Density (SLD) for the Materials Used in the Neutron Reflectivity Measurements

\begin{tabular}{lcc}
\hline \multicolumn{1}{c}{ materials } & density $\left(\mathrm{g} / \mathrm{cm}^{3}\right)$ & $\begin{array}{c}\text { neutron scattering length } \\
\text { density }(\mathrm{SLD})\left(10^{6} \AA^{-2}\right)\end{array}$ \\
\hline $\mathrm{Si}$ & 2.33 & 2.07 \\
$\mathrm{SiO}_{2}$ & 2.0 & 3.16 \\
$\mathrm{PVP}$ & 1.17 & 1.99 \\
$\mathrm{~d}-\mathrm{PS}$ & 1.12 & 6.4 \\
$\mathrm{~h}-\mathrm{PS}$ & 1.05 & 1.41 \\
toluene & 0.865 & 0.939 \\
d-toluene & 0.943 & 5.66 \\
cyclohexane & 0.779 & -0.278 \\
d-cyclohexane & 0.89 & 6.68
\end{tabular}

the polymer brushes assuming additivity of volumes. In the fitting, a model-independent multilayer approach is assumed in which layers of silicon, silicon oxide, polymer brush, and solvent are included. The SLD profile of the solvated polymer brush was modeled by a stack of slabs of varying SLD and thickness, while the other layers were modeled using a single SLD and thickness for each. The SLD values used for each material are listed in Table 2. The mean-square residual $\left(\chi^{2}\right)$ resulting from the fits were kept below 3 .

In most cases, the uniqueness of a fitted scattering length density (SLD) profile to a neutron reflectivity curve cannot be guaranteed. To verify the coherence of the fitted SLD profile to that of the brush, a self-consistent check is performed where the absorbed amount calculated from the fitted SLD is compared to that which is calculated from the thickness of the brush as measured by ellipsometry or X-ray reflectometry. In the present study, the dry layer thickness can be calculated from the density profile as $\sum_{i=1}^{n} \Delta Z_{i} \phi\left(Z_{i}\right)$, where $n$ is the number of slabs, $\Delta Z_{i}$ is the thickness of the $i$ th slab, and $\phi\left(Z_{i}\right)$ is the corresponding volume fraction of polymer in that slab. Only those fits to the reflectivity that are consistent with the ellipsometric or X-ray reflectivity dry layer thickness measurements are reported.

Even given these restrictions, there exist a narrow band of similar scattering length density profiles that will provide equivalent fits to the experimental data. Thus, the scattering length and copolymer density profiles provided in the following figures should be viewed as representative of this set of results, with the understanding that the deviation of this range of possible fits from this representative sample is small due to the mass balance restriction. It is important to emphasize that the band of density profiles that fit the experimental data is sufficiently narrow that it does not influence the physical interpretation of the results that follow.

X-ray Reflectivity Measurements. X-ray reflectivity measurements were carried out at ambient temperature at NIST to measure the dry layer thickness. A Bruker D8Advance diffractometer with $\mathrm{Cu} \mathrm{K} \alpha$ radiation was used. The wavelength $(\lambda)$ used is $1.5417 \AA$. Data collections were performed up to a wavevector $q=4 \pi \sin$ $\theta / \lambda=0.43 \AA^{-1}$, where $\theta$ is the incident angle.

\section{Results and Discussion}

Surface Density of Brushes. In order to properly determine the surface density of the brushes formed by preferential adsorption of the triblock copolymers, it is first appropriate to address the issue of whether the adsorbed chains are singly or 

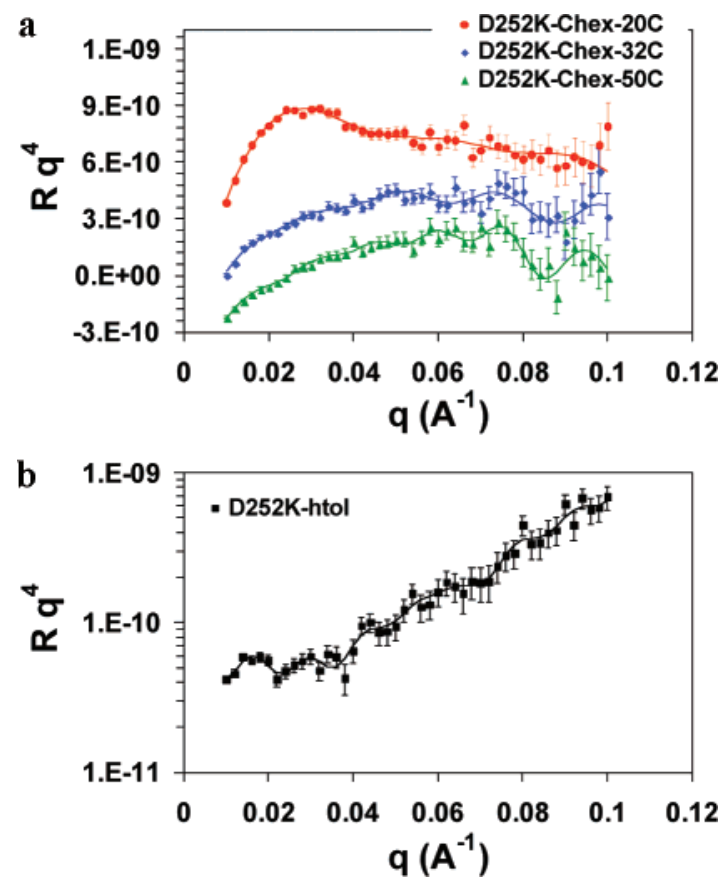

Figure 1. $R q^{4}$ vs $q$ plots for the D252K brush in (a) cyclohexane at $20{ }^{\circ} \mathrm{C}$ (red), $32{ }^{\circ} \mathrm{C}$ (blue), and $50{ }^{\circ} \mathrm{C}$ (green) and (b) in toluene at room temperature. For clarity, the curves in (a) have been shifted vertically. The best fits to the reflectivity are shown as solid lines.

doubly attached. Using the surface forces apparatus, Dai et al. ${ }^{21}$ found no bridging between an adsorbed PVP-PS-PVP layer and a bare mica surface, indicating the absence of dangling, singly bound chains. These results and previous work by us ${ }^{28}$ provide clear evidence for the preferential formation of PS loops at the toluene/substrate surface. Because one triblock copolymer chain can be considered equivalent to two diblock copolymers that are of half the length (molecular weight) of the PS block of the loop, the surface density, $\sigma$, can be calculated as

$$
\sigma=L_{\mathrm{dry}} N_{\mathrm{av}}\left(\frac{M_{\mathrm{PVP}}}{\rho_{\mathrm{PVP}}}+\frac{M_{\mathrm{PS}}}{\rho_{\mathrm{PS}}}\right)^{-1}
$$

Here, $L_{\mathrm{dry}}$ is the dry layer brush thickness measured by ellipsometry or X-ray reflectivity, $M_{\mathrm{PVP}}$ and $M_{\mathrm{PS}}$ are the molecular weights of their equivalent diblock counterparts, $N_{\mathrm{av}}$ is Avogadro's constant, and $\rho_{\mathrm{PVP}}$ and $\rho_{\mathrm{PS}}$ are the density for bulk PVP and d-PS or h-PS, respectively. $\rho_{\mathrm{PVP}}=1.17 \mathrm{~g} / \mathrm{cm}^{3}$, $\rho_{\mathrm{d}-\mathrm{PS}}=1.12 \mathrm{~g} / \mathrm{cm}^{3}$, and $\rho_{\mathrm{h}-\mathrm{PS}}=1.05 \mathrm{~g} / \mathrm{cm}^{3}$ are adopted in these calculations.

The reduced surface density, $\sigma^{*}$, characterizes the degree of overlap of the tethered chains, which directly influences the extent of stretching of the brushes:

$$
\sigma^{*}=\sigma / \sigma_{0}
$$

Here $\sigma_{0}=1 /\left(\pi R_{\mathrm{g}}{ }^{2}\right)$ is the surface density at overlap, above which neighboring chains begin to interact with each other. $R_{\mathrm{g}}$ $=0.29 M_{\mathrm{PS}}{ }^{0.5}$ was used to calculate the unperturbed radius of gyration of polystyrene (in nanometers) of the equivalent diblock in the melt. It is worth noting that basing $\sigma^{*}$ on the brush formed from the equivalent diblock copolymer ignores conformational differences that must exist between loops and singly tethered chains of half the molecular weight; regardless, eq 3 is useful because it captures, on average, the degree of overlap of the looped chains. Table 1 shows the thicknesses obtained from ellipsometry and X-ray reflectometry and the reduced surface density of the brushes studied here, which spans from ca. 3 to

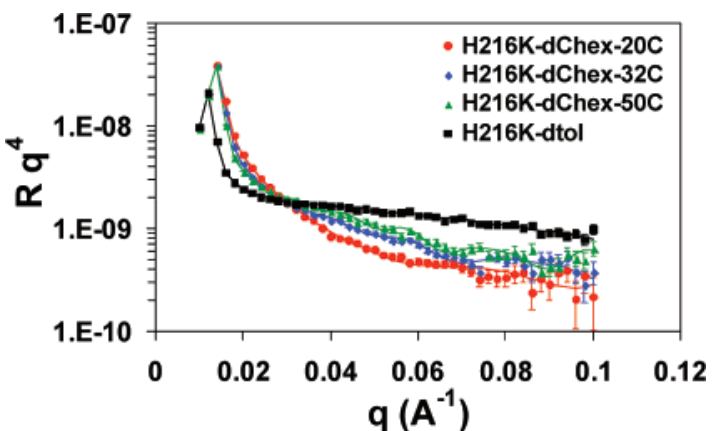

Figure 2. Comparison of the $R q^{4}$ vs $q$ plots for the brush of $\mathrm{H} 216 \mathrm{~K}$ in cyclohexane at $20{ }^{\circ} \mathrm{C}$ (red), $32{ }^{\circ} \mathrm{C}$ (blue), $50{ }^{\circ} \mathrm{C}$ (green), and in toluene (black), respectively. The best fits to the reflectivity profiles are again shown as solid lines.
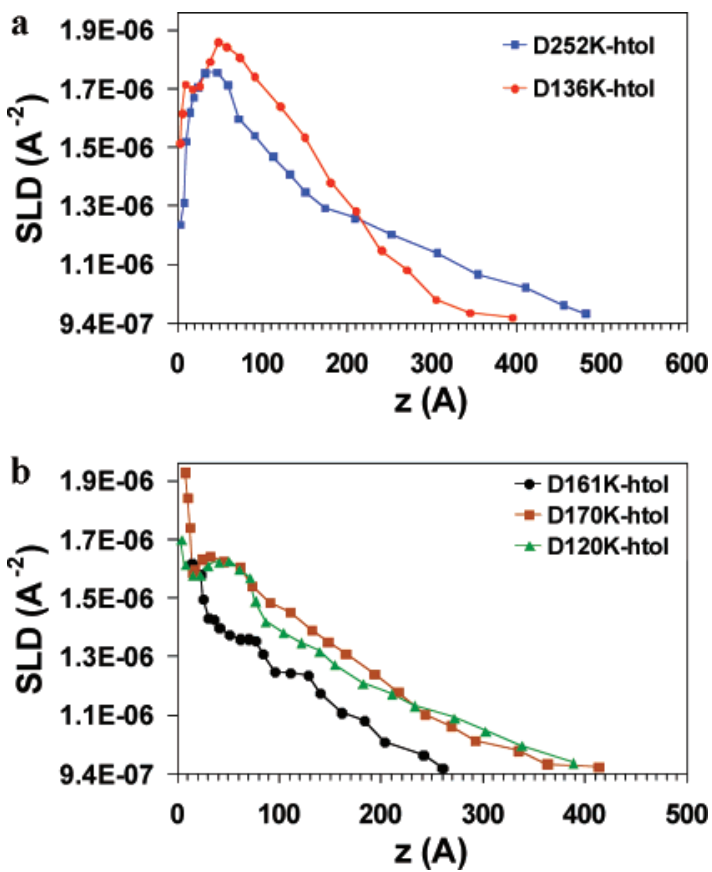

Figure 3. (a) SLD profiles for D136K (red) and D252K (blue) in toluene, where a lower SLD region near the interface is apparent. (b) SLD profiles for D120K (green), D170K, and D161K (black) in toluene show a region of higher SLD near the substrate-brush interface.

9, indicating that the layers formed exceed their overlap surface density; the segment density profiles presented later will show that all of the layers in toluene are stretched a few times their $R_{\mathrm{g}}$. Together these results reinforce the notion that these polymer amphiphihles have self-assembled into polymer brushes. ${ }^{28}$

Scattering Length Density Profiles. The reflectivity data were reduced and plotted as $R q^{4}$ as a function of $q$. The $R q^{4}$ vs $q$ plots for $\mathrm{D} 252 \mathrm{~K}$ and $\mathrm{H} 216 \mathrm{~K}$ are provided as examples in Figure 1 and Figure 2, respectively. As shown in these plots, the features of the reflectometry curves vary with solvent conditions. The systematic change in reflectivity with the solvent quality can be clearly seen for the hydrogenated PS brush in deuterated solvent, displayed in Figure 2. In cyclohexane, the reflectivity varies more sharply with $q$ at lower temperature, suggesting that the brush is denser and more uniform at low temperature. The fitted curves are also displayed in Figure 1 and Figure 2 as solid lines.

Figure 3a,b shows the scattering length density (SLD) profiles for all dPS looped layers in toluene obtained from fitting the reflectivity curves. Several features are noticeable in these figures. As shown in Figure 3a, the density profiles of the looped layers formed from D136K and D252K, which each have a 

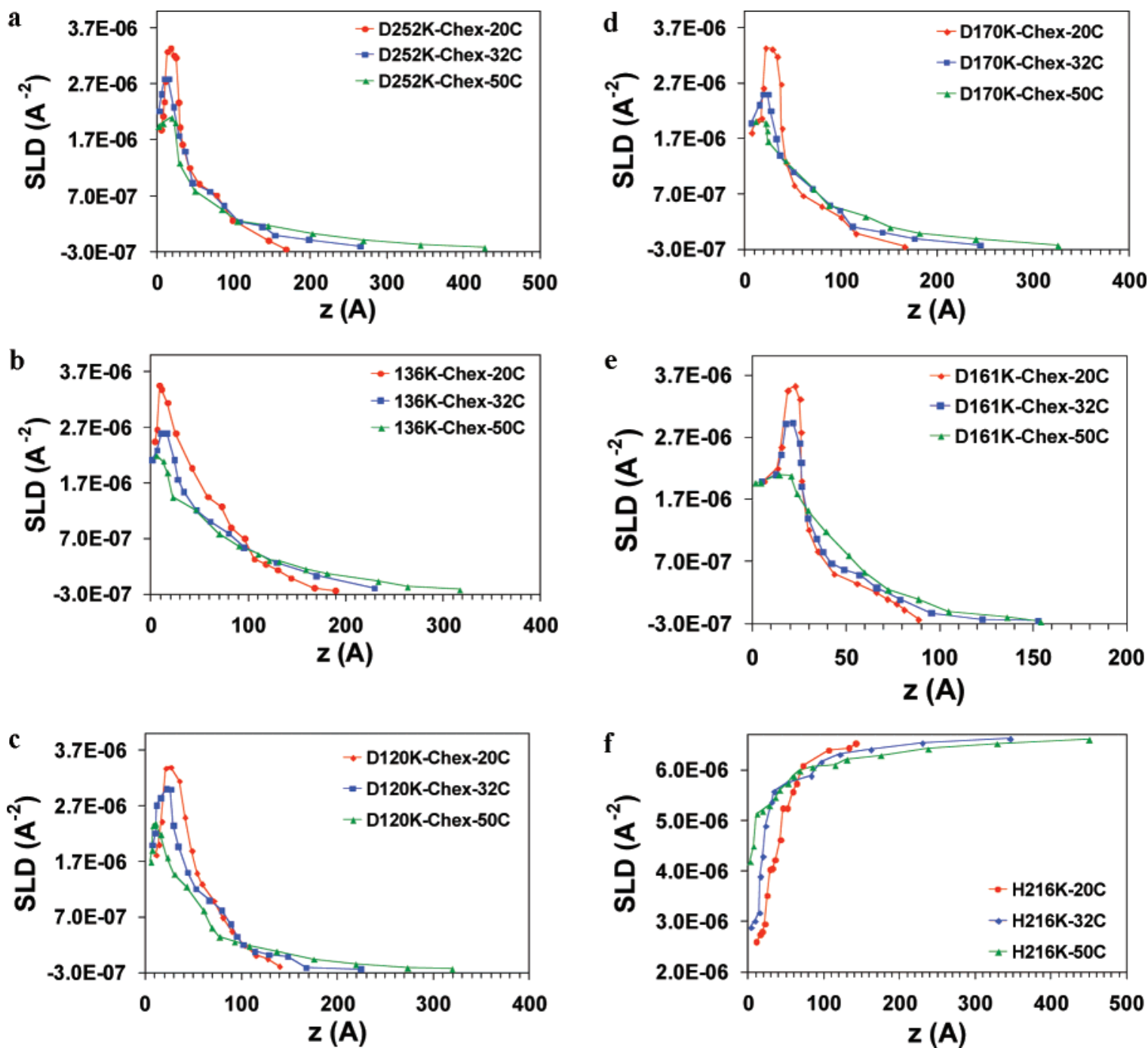

Figure 4. SLD profiles in cyclohexane for (a) D252K, (b) D136K, (c) D120K, (d) D170K, (e) D161K, and in deuterated cyclohexane for (f) $\mathrm{H} 216 \mathrm{~K}$ at $20^{\circ} \mathrm{C}$ (red), $32{ }^{\circ} \mathrm{C}$ (blue), and $50{ }^{\circ} \mathrm{C}$ (green).

styrene:vinylpyridine ratio of 10:1, show a maximum displaced from the surface by $\sim 30-50 \AA$. However, similar maxima are not observed in the SLD profiles of the D120K, D170K, and D161K samples, as shown in Figure 3b. Rather, a higher SLD near the substrate gives the best fit. The protonated PVP layer that is adsorbed on the silica surface should dominate the SLD near surface; however, the SLD in the near surface region is lower than that of pure PVP $\left(2 \times 10^{6} \AA^{-2}\right)$. Possible explanations for this observation include the partial swelling of the PVP by toluene and/or that the PVP blocks of the most asymmetric copolymers are unlikely to cover the entire surface, both of which have been reported previously. ${ }^{38}$ Differences in the shape of the SLD profile in the near-surface region are most likely due to the differences in the molecular asymmetry of the triblock, as the samples in Figure $3 \mathrm{~b}$ possess higher PVP fractions. In an attempt to resolve the structure of this nearsurface region, we also performed neutron reflectivity studies for PVP-dPS-PVP samples in deuterated toluene. In these systems, much larger contrast between the PVP and swollen dPS layer is expected. However, we were unable to obtain satisfactory density profiles for the PVP layers from these studies. This might be caused by the small thickness of this near-surface layer. At this point, a picture of the structure of this near-surface region remains unclear. This is unfortunate, as the elucidation of the near surface structure of these brushes may shed light on the existence of a depletion layer as suggested by theoretical ${ }^{1,2,14,35,39-41}$ and other experimental work. ${ }^{42-44}$

The scattering length density profiles of the brushes in cyclohexane under near- $\Theta$ solvent conditions are shown in Figure $4 \mathrm{a}-\mathrm{e}$. For the deuterated PS brushes in protonated cyclohexane, with increasing solvent quality, the overall brush thickness increases and the SLD near the surface decreases, indicating an increase of the degree of swelling of the brush with solvent quality, as expected.

Additionally, the best fits occur only when a region near the surface has an SLD close to that of PVP, indicating that the PVP, though swollen in toluene, is collapsed in cyclohexane. Moreover, the thicknesses of these layers seem to correlate with the fraction of PVP in the triblock copolymer: For the brushes made from copolymers with lower PVP content (D136K and D252K), the thickness of this anchoring layer was $\sim 10 \AA$, while thicker anchoring layers $(\sim 20 \AA)$ were obtained for the brushes with larger PVP content (D120K, D170K, and D161K).

Shape of the Density Profiles. The shape of the density profiles provides insight into the structure of the looped brushes, as was shown by previous work that examined the structure of singly bound PS brushes in toluene and cyclohexane. ${ }^{32}$ In that work, it was found that the majority of the density profile can be fit with a parabolic function, as shown in eq 1. Previous studies of brushes formed from triblock copolymers have 

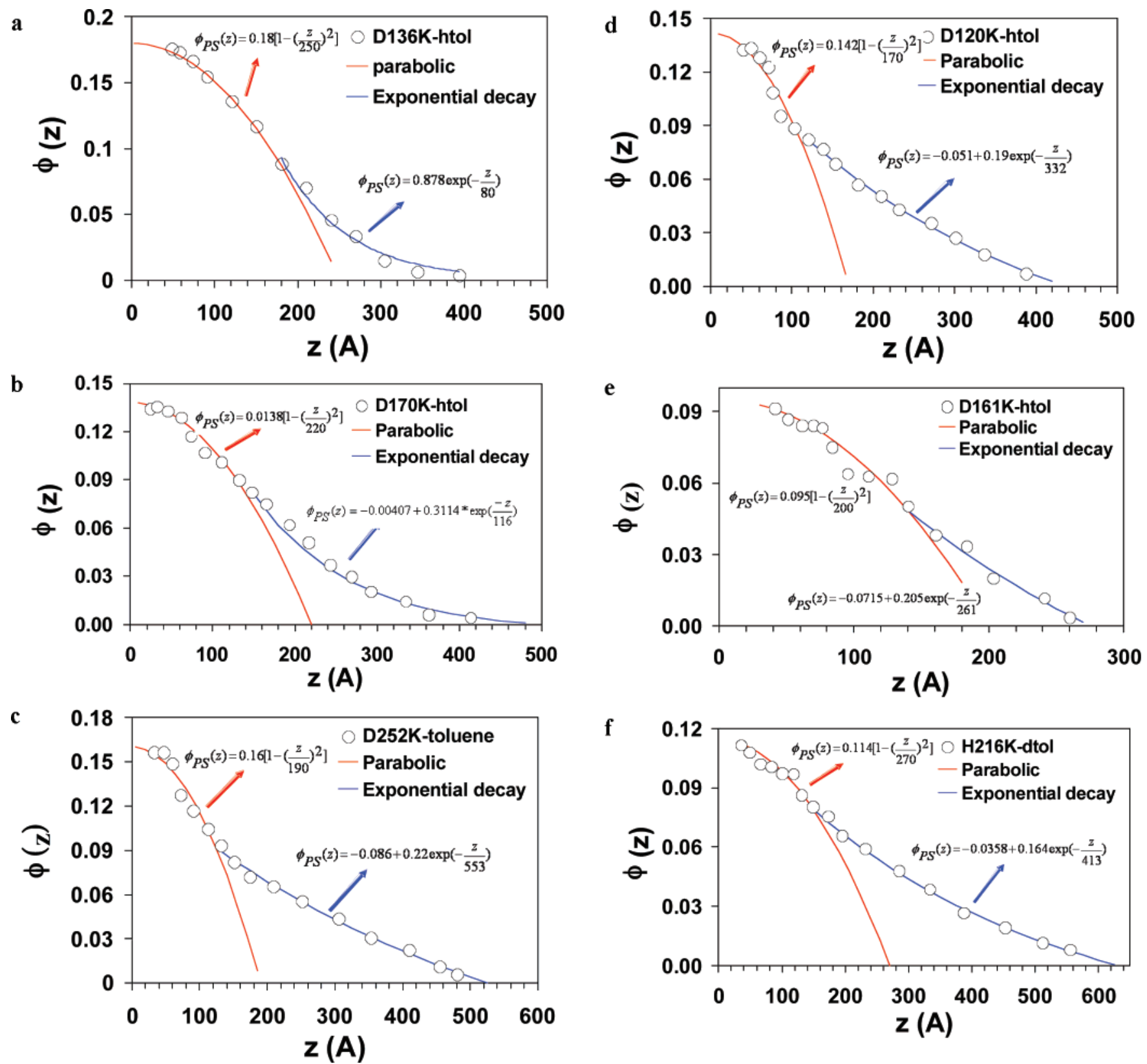

Figure 5. Density profiles in toluene for (a) D136K, (b) D170K, (c) D252K, (d) D120K, (e) D161K, and (f) H216K. All profiles can be fit with a parabolic function (red) plus an exponential decay (blue) as indicated in the plots.

indicated that they behave as brushes that are formed from diblock copolymers that are half the length of the triblock. ${ }^{28,45,46,47}$ However, the segment density profiles of the looped brushes studied here in the good solvent toluene (Figure $5 a-f)$ clearly show that these density profiles differ significantly from singly bound brushes: A parabolic function (eq 1) fits only a portion of these density profiles, and a long exponential tail is needed to model the density profile of these brushes. For the brushes in cyclohexane $\left(20,32\right.$, and $\left.50{ }^{\circ} \mathrm{C}\right)$, as shown in Figure 6, the segment density is also significantly different from that of the singly bound chains, since the density decays more rapidly as the brush extends away from the grafting surface, with no parabolic contribution. These density profiles can be described with an exponential decay, as shown in Figure 6 for D136K.

Thus, the density profiles of these doubly bound brushes in solvents of varying quality exhibit features that differ significantly from those of singly bound PS brushes under similar conditions. ${ }^{32}$ In toluene the parabolic region of the SLD profile is much narrower, and the extended exponential decay tail contributes more significantly to the profile than the profile of singly bound brushes. In cyclohexane, a parabolic region near the surface is not evident, and a long exponential decay dominates the profile.

Polydispersity of the brush chains can affect the parabolic shape of the density profile of a brush. ${ }^{48}$ The primary result of polydispersity in the polymer on the structure of the brush is a decrease in the parabolic region of the density profile and an increase in the extended exponential tail, as is observed in Figures 5 and 6. Rühe and co-workers consistently report that the segment density profile of polydisperse brushes in good solvents made by thermally initiated free radical polymerization are described by error functions. ${ }^{49-51}$ This is attributed to the polydispersity of the chains, which in these cases is $\sim 2$ based on size exclusion chromatography analysis of the polymer chains recovered from solution. In the present system, the molecular weight polydispersity of the triblock copolymers is in the range $1.1-1.2$, which should not be sufficient to create a brush where 


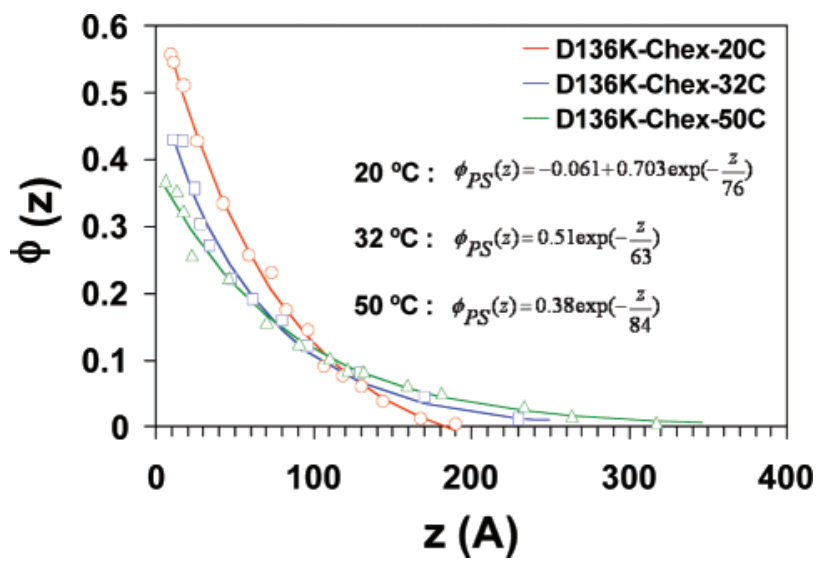

Figure 6. Density profiles for D136K in cyclohexane at $20^{\circ} \mathrm{C}$ (red), $32{ }^{\circ} \mathrm{C}$ (blue), and $50{ }^{\circ} \mathrm{C}$ (green). All curves can be fitted by an exponential decay as indicated in the plot.

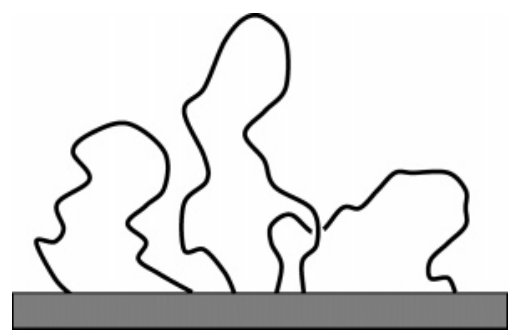

Figure 7. Schematic representation illustrating how variations in distances between tethering points and chain crossover affect the ability of the tethered loops to extend normal to the surface. The net result is that the layer appears to be comprised of loops of different sizes; this effect is referred to as an "anchor-induced" polydispersity.

the profile is dominated by the exponential tail. However, in these doubly bound brushes, a different form of polydispersity may exist that will impact the density profile of the looped brush-namely, a distribution of distances between the two anchored PVP blocks of a single chain. As shown schematically in Figure 7, the extent to which a given chain can extend away from the surface depends on the distance between its two PVP anchor points. Thus, chains that have both tethering blocks in close proximity may extend further away from the surface, but the extension of those chains whose ends are more separated will be more restricted. Also, because loop formation does not require both PVP end blocks to arrive at the surface at the same instant, some loops may arch over other loops, thereby pinning portions of one loop below another. The impact of these forms of "anchor induced" polydispersity on the density profile is a less uniform extension of the dPS loops away from the surface among the chains and a brush with more dilute regions at the edge of brushes, which will manifest in the density profiles as a narrowing in the parabolic region and expansion in the exponentially decaying tail, as is observed in these studies. It is important to emphasize that this behavior is a unique feature for the "looped" brush and may play an important role in determining the interfacial behavior of these novel brushes.

This polydispersity and resultant gradually decaying density profile suggests that with doubly bound brushes one may be able to modify interfacial properties in ways not possible with singly bound brushes that are one-half the length. Because the solvated looped layers are more dilute at the periphery, intersurface interactions (forces) governed by steric repulsion are likely to be "softer", similar to the case of asymmetric bimodal brushes made from $\mathrm{ABC}$ triblock copolymers preferentially adsorbed through the B block. ${ }^{52}$ The dynamic structure of these dilute looped layers, which has been implicated as playing a significant role in the frictional interactions between solvated brushes ${ }^{53}$ may also be altered because of extended, dilute periphery. Experimental studies to test this hypothesis can provide additional insight into the behavior of these unique systems. Additionally, invoking the structure shown in Figure 7 agrees with previous work $^{54}$ that has demonstrated that multiblock copolymers enhance the adhesion across polymerpolymer interfaces relative to a diblock copolymer, apparently because it is more difficult to disengage penetrating chains that cross the interface and entangle in the loops.

Thus, these careful experiments provide, for the first time, the detailed structure of doubly bound polymer brushes indicating a relatively diffuse density profile, which will play an important role in the interfacial behavior of these and similar systems.

Swelling Behavior in Near- $\Theta$ Solvents. To quantify the swelling behavior of the doubly bound brush as a function of solvent quality, the height of an equivalent AlexanderdeGennes (steplike) brush, $d^{*}$, is calculated as suggested by Habicht et al. ${ }^{45}$ from the first moment of the segment density profile:

$$
d^{*}=\frac{2 \int_{0}^{\infty} z \phi(z) \mathrm{d} z}{\int_{0}^{\infty} \phi(z) \mathrm{d} z}
$$

In the vicinity of the $\theta$ temperature where the second virial is taken to vary linearly with temperature, a reduced temperature $\tau=(T-\theta) / T$ can be used to describe the solvent quality. In this expression $T$ is the experimental temperature and $\theta$ is the $\theta$ temperature for the dPS - cyclohexane system, which we take to be $32{ }^{\circ} \mathrm{C}$. (It should be noted that the $\theta$ temperature for dPS in cyclohexane has been determined to be $30{ }^{\circ} \mathrm{C}, 55$ and for hydrogenated PS in deuterated cyclohexane, $\theta$ temperatures of $38.6^{56}$ and $40{ }^{\circ} \mathrm{C}^{51}$ have been reported, depending on molecular weight.)

Figure 8a shows how temperature, and thus solvent quality, affects the swelling of the looped polymer brushes in cyclohexane. In this figure, a normalized thickness, $d^{*} / d^{*}(\theta)$, where $d^{*}(\theta)$ is the thickness at the $\theta$ condition, is used. The general trend displayed in Figure $8 \mathrm{a}$ is that less-dense looped layers change their (average) thickness more than denser looped layers as solvent quality is changed, suggesting that brushes of lower surface density are able to adjust their structure more significantly in response to changes in environmental conditions. This trend is shown more clearly in Figure $8 b$, where the differences in the normalized thickness between 20 and $50{ }^{\circ} \mathrm{C}$ are plotted as a function of the surface density. The outlier in this data set is the D $161 \mathrm{~K}$ sample, which is a symmetric triblock and the least overlapped brush $\left(\sigma^{*}=2.9\right.$, as shown in Table 1).

Karim et al. previously showed that the swelling behavior of end-tethered polymer brushes (singly tethered layers) bears a resemblance to that of lightly cross-linked polymer gels. ${ }^{57}$ In this analogy, brushes are viewed as a gel having their crosslinks at the solid surface, and changes in solvent quality induce a swelling response in the dimension of the brush normal to the tethering surface. Using two dimensionless variables-a scaled temperature and degree-of-swelling (a scaled concentration) derived by Zrinyi and Horkay ${ }^{58}$ using a Flory approach to describe the equilibrium swelling response of a gel-Karim et al. showed that the swelling behavior of relatively dense, singly tethered PS brushes in cyclohexane around the $\theta$ temperature fall upon a master curve obtained from studies of poly(vinyl acetate) gels of different cross-link density in isopropyl alco- 

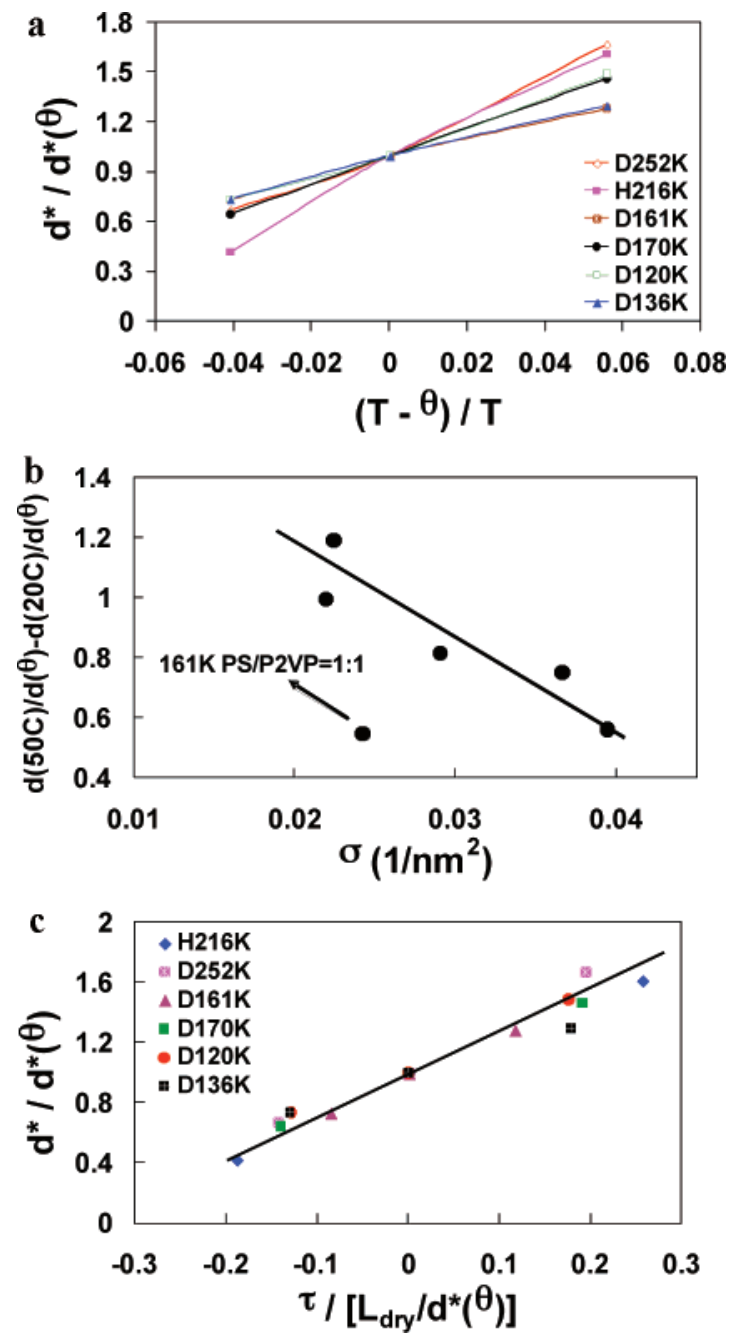

Figure 8. (a) Normalized average thickness as a function of the normalized temperature. (b) Difference in the normalized average thickness at 20 and $50{ }^{\circ} \mathrm{C}$ as a function of the surface density. (c) Master curve for the normalized brush thickness as a function of normalized temperature, further reduced by a volume expansion factor of the brush under near- $\Theta$ conditions relative to the dry brush, $\phi(\theta)=L_{\mathrm{dry}} / d(\theta)$.

hol. ${ }^{53,54}$ Following these treatments, we plot in Figure $8 \mathrm{c}$ the degree of swelling, $d^{*} / d^{*}(\theta)$, vs the $\tau / \phi(\theta)$, where $\phi(\theta)$, defined as $L_{\mathrm{dry}} / d(\theta)$, takes into account differences in "cross-link density" (grafting density and its implication on the volume expansivity) of the various looped brushes. Clearly, this scaling is more appropriate than that in Figure $8 \mathrm{~b}$ to physically account for the parameters that control the expansion of the brush with a change in solvent quality. As observed from Figure 8c, the results for the various looped brushes coalesce to a single curve, reinforcing the brush-gel analogy. On one hand, this result is not unexpected: we $\mathrm{e}^{28}$ and others ${ }^{33,48}$ have previously shown that the average equilibrium structure (i.e., thickness or height) of looped brushes can be represented by an "equivalent diblock" model. However, it is interesting that the segment density profiles of these sparsely tethered looped layers are dramatically different from the more dense singly tethered brush layers examined by Karim et al. ${ }^{32}$ and yet respond similarly to an external stimulus, indicating that the swelling behavior is governed more so by the tethering of the chains to the surface rather than the details of the segment density profile normal to the tethering surface.

\section{Conclusions}

Neutron reflectivity measurements provide the detailed density profile of looped PVP-PS-PVP brushes under varying solvent conditions. While previous results prove that the average size of such brushes is similar to those found in singly bound brushes that are half the length, these more detailed studies indicate that their structure is dominated by a diffuse, extended periphery best described by an exponential decay. This exponential decay in the density normal to the surface satisfactorily describes the density profile in near- $\Theta$ conditions and remains dominant in a good solvent. The characteristics of these profiles appears to be intrinsically related to the self-assembly of loops, which provides an additional polydispersity-like effect due to variations in the distances between tethering sites of the doubly bound chains and the potential for some loops to crossover other loops. This novel density profile may impact the interfacial properties of multiply bound chains and must be considered when analyzing such systems.

While the swelling response of the looped layers can be adequately captured by an average (mean-field) representation, and this representation strengthens further the brush-gel analogy, future design of polymer-modified interfaces is likely to profit from an understanding of how chain architecture, including connectivity and asymmetry, can be used as a tool to alter nanoscale structure and therefore interfacial properties.

Acknowledgment. This work was financially supported by the National Science Foundation through its Collaborative Research in Chemistry Program (CRC-CHEM 0304807). We acknowledge the support of the National Institute of Standards and Technology, U.S. Department of Commerce, in providing the neutron research facilities used in this work. The assistance from Drs. Sushil K. Satija and Saibal Basu at NIST is greatly appreciated. Z.H. and H.J. also thank the grant from UMCP/ NIST Neutron Outreach program. S.M.K.II acknowledges with thanks the stimulating environment of the Center for Nanophase Materials Sciences at Oak Ridge National Laboratory, which provided for the completion of this work.

\section{References and Notes}

(1) Alexander, S. J. Phys. (Paris) 1977, 38, 977.

(2) de Gennes, P. G. Macromolecules 1980, 13, 1069

(3) Milner, S. T. Science 1991, 251, 905.

(4) Milner, S. T.; Witten, T. A.; Cates, M. E. Europhys. Lett. 1988, 5, 413.

(5) Milner, S. T.; Witten, T. A.; Cates, M. E. Macromolecules 1988, 21 , 2610.

(6) Halperin, A.; Tirrell, M.; Lodge, T. P. Adv. Polym. Sci. 1992, 100, 31.

(7) Patel, S.; Tirrell, M. Annu. Rev. Phys. Chem. 1989, 40, 597.

(8) Napper, D. H. Polymeric Stabilization of Colloidal Dispersions; Academic Press: London, 1983.

(9) Smith, J. W.; Kramer, E. J.; Xiao, F.; Hui, J.; Reichharts, W.; Brown, H. J. Mater. Sci. 1993, 28, 4234.

(10) Smith, J. W.; Kramer, E. J.; Mills, P. J. J. Polym. Sci., Polym. Phys. 1994, 32, 1731

(11) Derulle, M.; Tirrell, M.; Marciano, Y.; Hervet, H.; Léger, L. Faraday Discuss. 1994, 98, 55.

(12) Brown, H. R. Faraday Discuss. 1994, 98, 47.

(13) Munch, M. R.; Gast, A. P. Macromolecules 1990, 23, 2313.

(14) Toomey, R.; Mays, J.; Tirrell, M. Macromolecules 2004, 37, 905.

(15) Webber, R. M.; Anderson, J. L. Langmuir 1994, 10, 3156.

(16) Belder, G. F.; ten Brinke, G.; Hadziioannou, G. Langmuir 1997, 13, 4102.

(17) Webber, R. M.; Anderson, J. L.; Jhon, M. S. Macromolecules 1990, 23, 1026.

(18) Field, J. B.; Toprakcioglu, C.; Dai, L.; Hadziioannou, G.; Smith, G.; Hamilton, W. J. Phys. II 1992, 2, 2221.

(19) Satija, S. K.; Majkrzak, C. F.; Russell, T. P.; Sinha, S. K.; Sirota, E. B.; Hughes, G. J. Macromolecules 1990, 23, 3860.

(20) Anastassopoulos, D. L.; Vradis, A. A.; Toprakcioglu, C.; Smith, G. S.; Dai, L. Macromolecules 1998, 31, 9369.

(21) Dai, L. M.; Toprakcioglu, C.; Hadziioannou, G. Macromolecules 1995, $28,5512$.

(22) Ligoure, C. Macromolecules 1991, 24, 2968. 
(23) Blum, F. D.; Sinha, B. R.; Schwab, F. C. Macromolecules 1990, 23, 3592

(24) Tian, P.; Uhrig, D.; Mays, J. W.; Watanabe, H.; Kilbey, S. M. II Macromolecules 2005, 38, 2524.

(25) Shull, K. R. Faraday Discuss. 1994, 98, 203

(26) Irvine, D. J.; Mayes, A. M.; Griffith-Cima, L. Macromolecules 1996, 29, 6037.

(27) Irvine, D. J.; Mayes, A. M.; Satija, S. K.; Barker, J. G.; Sofia-Allgor, S. J.; Griffith, L. G. J. Biomed. Mater. Res. 1994, 28, 329.

(28) Alonzo, J.; Huang, Z. Y.; Liu, M.; Mays, J. W.; Dadmun, M. D.; Kilbey, S. M. II Macromolecules 2006, 39, 8434.

(29) Shim, D. F. K.; Cates, M. E. J. Phys. (Paris) 1989, 50, 3535.

(30) Zhulina, E. B.; Borisov, O. V.; Pryamitsym, V. A.; Birshtein, T. M. Macromolecules 1991, 24, 140.

(31) Perahia, D.; Wiesler, D. G.; Satija, S. K.; Fetters, L. J.; Sinha, S. K.; Milner, S. T. Phys. Rev. Lett. 1994, 72, 100.

(32) Karim, A.; Satija, S. K.; Douglas, J. F.; Ankner, J. F.; Fetters, L. J. Phys. Rev. Lett. 1994, 73, 3407.

(33) Chen, C.; Dan, N.; Dhoot, S.; Tirrell, M.; Mays, J.; Watanabe, H. Isr. J. Chem. 1995, 35, 41 .

(34) Lai, P. Y.; Binder, K. J. Chem. Phys. 1992, 97, 586.

(35) Grest, G. S.; Murat, M. Macromolecules 1993, 26, 3108.

(36) Balsara, N. P.; Tirrell, M.; Lodge, T. P. Macromolecules 1991, 24, 1975-1986.

(37) http://www.ncnr.nist.gov/programs/reflect/data_reduction/software/ index.html.

(38) Parsonage, E.; Tirrell, M.; Watanabe, H.; Nuzzo, R. G. Macromolecules 1991, 24, 1987.

(39) Whitmore, M. D.; Noolandi, J. Macromolecules 1990, 23, 3321

(40) Muthukumar, M.; Ho, J. S. Macromolecules 1989, 22, 965.

(41) Wang, W.; Lin, Z. Z.; Ebner, C.; Nikas, Y. Phys. Rev. E 1993, 48, 4622 .
(42) Cosgrove, T.; Heath, T. G.; Phipps, J. S.; Richardson, R. M. Macromolecules 1991, 24, 94

(43) Cosgrove, T.; Phipps, J. S.; Richardson, R. M.; Hair, M. L.; Guzonas, D. A. Macromolecules 1993, 26, 4363.

(44) Kent, M. S.; Lee, L. T.; Factors, B. J.; Rondelez, F.; Smith, G. S. J. Chem. Phys. 1995, 103, 2320.

(45) Dai, L.; Toprakcioglu, C. Macromolecules 1992, 25, 6000.

(46) Tirrell, M.; Patel, S.; Hadziiaonnou, G. Proc. Natl. Acad. Sci. U.S.A. 1987, 84, 4725

(47) Siqueira, D. F.; Stamm, M.; Breiner, U.; Stadler, R. Polymer 1995, $36,3229$.

(48) Milner, S. T.; Witten, T. A.; Cates, M. E. Macromolecules 1989, 22, 853.

(49) Habicht, J.; Schmidt, M.; Rühe, J.; Johannsmann, D. Langmuir 1999, $15,2460$.

(50) Biesalski, M.; Rühe, J. Macromolecules 2002, 35, 499.

(51) Prucker, O.; Rühe, J. Langmuir 1998, 14, 6893.

(52) Dhoot, S.; Watanabe, H.; Tirrell, M. Colloids Surf., A 1994, 86,

(53) Forster, A. M.; Mays, J. W.; Kilbey, S. M. II J. Polym. Sci., Part B: Polym. Phys. 2006, 44, 649.

(54) Eastwood, E.; Dadmun, M. D. Macromolecules 2002, 35, 5069-5077.

(55) Strazielle, C.; Benoit, H. Macromolecules 1975, 8, 203.

(56) Siporska, A.; Szydlowski, J.; Rebelo, L. P. N. Phys. Chem. Chem. Phys. 2003, 5, 2996.

(57) Karim, A.; Douglas, J. F.; Horkay, F.; Fetters, L. J.; Satija, S. K. Physica B 1996, 221, 331.

(58) Zrinyi, M.; Horkay, F. Macromolecules 1989, 22, 1306.

MA702179X 